\title{
The Paradoxes of PrEP: Rejection, Reluctancy, and Novel Gay Identities in Biomedical HIV Prevention in Serbia
}

https://doi.org/10.51897/interalia/ODVI6514

\author{
Zoran Milosavljević \\ Serbian Academy of Arts and Sciences \\ ORCID: 0000-0001-6098-5110
}

\begin{abstract}
This article explores the different ways in which gay men in Serbia perceive PrEP as a novel method of HIV prevention. In the article, I draw on data from my research on PrEP use among thirty gay men in Belgrade. The use of PrEP is still very low amongst gay communities in Serbia due to their rejection of PrEP and due to the stigma around PrEP use. In Serbia, the social significance of PrEP relates to HIV status disclosure on gay social/dating media. Paradoxically, on gay dating sites, the signifier "PrEP" blurs the line between HIV positive gay men - who have achieved undetectable HIV status through a potent ARV therapy - and those HIV negative gay men who use PrEP as a preventative tool against HIV transmission. In the article, I will argue that a new form of gay identity has emerged on gay dating apps in Serbia - "undetectable, on PrEP." This new identity emerges from confusion in HIV risk assessment. The use of PrEP has been seen as a marker to denote someone's HIV negative status and to protect them from HIV transmission. However, some gay men with an undetectable HIV status would like to be regarded as HIV negative even though they are not, and thus they use the signifier "On PrEP" to highlight their desire to claim an HIV negative status. PrEP has many symbolic valences: from HIV status disclosure to assumed promiscuity. As I will argue, while the health paradigm is of utmost importance for Serbian gay men, internalized stigma additionally drives the low uptake of PrEP amongst gay communities in Serbia, thus contributing to the confusion regarding PrEP use and the overall approach to HIV prevention. This article finds that those respondents who accept PrEP without stigma or confusion regarding their HIV status are also more willing and ready to recommend using PrEP to other gay men.
\end{abstract}

Keywords: gay men, PrEP, Serbia, HIV status, HIV prevention

\section{Introduction}

The use of PrEP in Serbia among gay men for HIV prevention is a complex question, best defined through ambivalence and reluctance. There is a high level of confusion among gay men on the role of PrEP, which leads to a certain paradox. First, this potent preventive tool cannot fulfil its preventive role against HIV transmission because so many in the gay community either reject PrEP outright or are reluctant to use it; and second, in many cases its potency has been "hijacked" by the fear of stigmatization and confusion about for whom PrEP is designed - whether for HIV negative or HIV positive gay men. In this confusion around PrEP and HIV status a new sexual identity - "undetectable, on PrEP" - emerges with further risk assessment implications.

This article contributes to the current debate on the social signification of PrEP (Auerbach and Hoppe, 2015; Calabrese and Underhill, 2015; Dean, 2015; Eaton et al., 2017; Castro, Delabre and Molina, 2019; Nicholls and Rosengarthen, 2019; Gomez et al., 2020). The meaning of PrEP as a preventive method 
against HIV transmission goes beyond simple "pill-popping," and as Auerbach and Hoppe cogently noted, "PrEP embodies a range of interacting physiological, psychological and social realities that together affect not only an individual's risk of avoidance of HIV infection but also relationship dynamics, sexual cultures and social arrangements that have influence beyond HIV" (2015: 30). The gay community's response to PrEP is also being driven by a broader social signification of HIV status; one that puts questions of risk and responsibility at the heart of HIV prevention (Jaspal and Daramilas, 2016; Thomann, 2018; Hildebrandt, Bode et Ng, 2019). The match between objective risk and subjective perception of risk among gay men is frequently a mismatch and it erodes PrEP's preventive potential. Race (2016) sees PrEP as a signifier to the changing paradigm of HIV prevention and a "reluctant object" to be or not to be used in gay sexual practices.

The relation between PrEP and HIV status goes beyond a simple division between HIV negative and HIV positive gay men and their opinions about risk assessment, which has even resulted in the introduction of new categories of identification, such as the "preventionist identity" (Calabrese and Underhill, 2015) or "HIV negative, on PrEP" (Thomann, 2018). Brennan (2017) describes how gay pornography has changed under the influence of PrEP, into expositions of the full condomless or "bareback" sex act, and he underlines the fact that this situation has further implications for gay sexual practices. Golub, Gamarel and Surace (2017) report that PrEP users are sometimes perceived as HIV positive and deemed to be lying about their HIV status. Klein and Washington (2020) report the lack of interest in learning about PrEP and problems in its adoption among a portion of gay men in the US.

\section{PrEP in Serbia}

The HIV/AIDS epidemic in Serbia is an unstable one. Serbia is a low prevalence country, but the number of cases per year is on the rise, mostly among the MSM population (UNAIDS, 2020). Since 2000 MSM have been the predominant group among newly registered cases, with $80 \%$ of all registered cases in 2019 (IOPHOS, 2020). The antiretroviral therapy (ARV) has been available since 1997, and $64 \%$ of HIV positive individuals were on ARV in 2019 (IOPHOS, 2020).

PrEP in the form of Truvada was registered in Serbia in 2015 by the Agency of Medicines and Medical Devices of Serbia (ALIMS). Initially, the major impediment to its distribution was the price of 30,000 dinars (approx. 250 euros monthly) as this was simply too expensive for the majority of gay men. The first step toward better access to PrEP was the registration of the generic drug Gilestra Duo T, produced by the pharma company Actavis. It was registered in 2018 and offered the treatment at a much lower price of 50 euros per box/monthly therapy. Given the fact that the average salary in Serbia as of July 2021 is approximately 550 euros (Statistical Office of the Republic of Serbia, 2021), even the generic form of PrEP remains unaffordable for many.

The semi-official start of PrEP in Serbia was in August 2019. The protocol for prescribing PrEP was drawn up by the Ministry of Health in 2019, in consultation with the People Living With HIV/AIDS 
(PLWH) non-governmental organizations. This protocol stated that the use of PrEP should be commenced only after the potential recipient of PrEP has first undegone HIV/STD testing. After this initial testing and start of PrEP, testing for HIV and STDs should be done every three months as a method of screening. STD screening is a form of epidemiological surveillance but also a signifier of successful combination of preventive measures in use - the conventional prevention through condom use, which protects against other STDs, and the biomedical prevention of PrEP, which protects against HIV transmission but not against other STDs.

When one is screened to be HIV negative, they can get the so-called precept (i.e., an informal prescription) from an HIV/AIDS specialist in the Clinic for Infectious Diseases in Belgrade. With this prescription for PrEP, one can then buy the drug in the pharmacy close to the Clinic. Nevertheless, many gay men in Serbia are using "informal" or "wild" PrEP, the source of which is virtually impossible to establish, for it is usually imported and sold on the black market. Brisson (2017) warns that if public health officials interfere with the "informal use" of PrEP among gay men and MSM, they aggravate the problem because such "informal use" contributes to HIV risk-reduction. The medical professionals' counterargument is that "informal use" of PrEP could lead to HIV viral resistance to ARV due to frequent nonadherence. When the adherence to PrEP is not respected, the concentration of ARV in the blood stream is insufficient to stop the replication of the HIV virus and there is a possibility of further spread of HIV after unsafe sexual practices.

The ongoing talks between the Ministry of Health, the Public Health Insurance Fund, medical specialists and gay community members who are organized in gay and PLWH non-governmental organizations, should eventually result in the broader availability of PrEP. Although PrEP is supposed to be subsidized and gradually made available throughout Serbia, by the autumn of 2020, nothing had changed in Serbia regarding PrEP use and PrEP recommendations. The 2020 Covid-19 pandemic has indisputably contributed to the impediment of PrEP use because access to preventive services and medical specialists has been disrupted for the majority of gay men.

\section{Methodology}

This paper is based on qualitative research carried out through interviews. I conducted 30 semistructured interviews. 24 gay men were randomly sampled when they attended a gay club in Belgrade in the period between $7^{\text {th }}$ October and $29^{\text {th }}$ December 2019. 6 gay men were sampled by the "snowball" technique. All of them identified as gay men during the interview and they gave their verbal informed consent before the start of the interview. The HIV status of the participants was not the dominant factor in the recruitment process, since the aim of the research was to map the overall situated knowledge about PrEP in the gay community, regardless of individual HIV status. 
The interviews were coded and, subsequently, the codes were grouped into categories and themes. I employed thematic and discourse analyses for the data set to be analyzed. Specifically, in this article I tend to focus on two emerging themes from my research - first, PrEP use in relation to sexual sociability and a new sex/health identity; and second, the implications of stigma reproduction.

\section{PrEP use in relation to sexual sociability and a new sex/health identity}

In discussions of the potential use of PrEP, there are many aspects of HIV discourse on prevention which tend to shape PrEP's acceptance and good preventive results. Some of the most important are: access to PrEP, which depends on the cost and availability; and adherence to PrEP, defined as the discipline to take the daily dosage regularly and to respect suggested protocols of use. All other characteristics of PrEP prevention could be derived from these two main ones. Race draws attention to these interconnected aspects of PrEP:

The issues of nonadherence, risk compensation, cost, access, unwanted toxicity, and the possible development of resistant viruses in the context of undetected seroconversion and suboptimal treatment (which is what PrEP would be in these circumstances) are real and must be addressed. (2016: 17-18)

Such a complex interconnection of aspects related to PrEP shows that an individual decision to use PrEP depends on many factors. For instance, 14 of my interviewees reported that they would gladly use PrEP; 10 reported that they would not use it under any circumstances; while 6 were not sure whether they would like to be on PrEP or not. This last group justified the ambiguity of their position in relation to PrEP by stating that they did not feel as if they had sufficient information about it.

The tension between PrEP users and non-users with regard to stigmatization, which I discuss in the next part of this paper, also poses the question of risk and responsibility among and between gay men, which lies at the heart of the debate about PrEP. For gay men in Serbia, the overall scarce use of PrEP leads to various ambiguities and interpretations of the role of PrEP in HIV prevention and risk management, thus making it a proper "reluctant object" (Race, 2016: 17). Serbian gay men are also reluctant to reach for PrEP because they do not know whether PrEP is used for HIV prevention (only by HIV negative gay men) or for HIV viral load control (only by HIV positive men). HIV positive gay men use a combination of ARV for HIV treatment that is the same as PrEP, so some HIV positive gay men use the signifier "PrEP" as a proof that they actually are in the same position as HIV negative gay men.

With such confusion regarding PrEP in Serbia, the risk assessment and responsibility in relation to HIV transmission are defined by the biomedical conception of PrEP as a tool of protection, rather than a bestowal of permission for the deregulation of sex. In the discussion presented by Dean, PrEP is seen through the lens of "sexual and morale failure," and he notices as follows: 
For gay men to identify themselves as 'at risk' entails an acknowledgement of their desire for raw sex that goes against community norms. To acknowledge this desire is potentially a risk in itself, because it compromises our image of the responsible gay man who always practices safer sex. To inquire about Truvada for PrEP may be felt as a sign of failure, or a confession that one wishes to behave in a way that the mainstream gay community has coded as immoral (2015: 229-230).

PrEP's proven potential is often eroded by the community's perception of it as an unreliable tool of protection for many gay men and as "a new form of prevention" for others. The negative side effects of the use of PrEP worry some gay men. For instance, a 42-year-old teacher from Čačak/Central Serbia, who is worried that PrEP is a product with "high toxicity," explains:

I might consider taking PrEP in 5-6 years' time [...] Maybe, I'd do it! I would use it only as the last resort against HIV. It seems to me that it's "pure poison."

(Anonymous, 42-year-old)

This discursive notion of PrEP as a "poison" has had the effect of eroding trust in PrEP and therefore limiting its success in the at-risk demographic in Serbia that is comprised by the gay community. More positive experiences with PrEP and then the sharing of the testimony of those positive experiences among gay men are certainly needed in order to achieve a significant improvement in $\operatorname{PrEP}$ uptake.

Conspiracy theories are another factor negatively influencing PrEP's introduction in Serbia. One of these is that PrEP is a product of a corrupt large profit-driven pharmaceutical company, thus not medically or scientifically reliable for everyday use by gay men. Serbia is not alone in this respect. In the context of the USA, Eaton et al. (2017) report on the conspiracy-related theory of PrEP rejection among gay men in the south. What is specific for Serbia is that such an attitude persists among a large number of gay men, and it contributes to the spreading of false claims that there is a bigger conspiracy theory behind PrEP whose origin is in the "pharmaceuticalisation" of gay identity, in other words, a scheme to form the gay experience through medication instead of sexual and homosocial practices. In Serbia, this issue is only an extension of the discourse around the biomedicalization and pharmaceuticalisation of gay health, where there is a cluster of theories related to PrEP, all of which revolve around Big Pharma-related conspiracies, fake news, and the theories about the deliberate concealment of public information.

Yet another attribute of PrEP influencing the level of its uptake is the perception of PrEP as a "novelty": i.e. something newly available with which to experiment on gay men's health. Although this is not a dominant discourse, it nevertheless drives some gay men and MSM to reject PrEP as a "still to be proven" solution for the prevention of HIV infection, and this takes time. With this perception of PrEP as a "novel" drug, additional efforts are and will continue to be necessary to introduce PrEP to a 
broader gay audience if the level of its uptake and impact are to be raised. The testimony of Aleksandar2, a 33-year-old artist from Belgrade, is germane here. He has also been alerted about PrEP's side effects, for he states:

I wouldn't take PrEP because it is still under-researched, especially in terms of its side effects.

(Aleksandar2, 33-year-old)

For Aleksandar2, PrEP is a novelty - an under-researched medicine and treatment regime - and that is the main reason that he would not consider taking PrEP in this initial phase of its introduction in Serbia. Nikola, a 30-year-old lawyer, has reservations about PrEP mostly related to his fear that it could destabilize his management of the chronic illness he already suffers from, and he explains:

I would consider taking PrEP if it did not interfere with my chronic disease. I have irritable bowel syndrome, and I've also heard that it fucks you in the head. One of my friends was on PrEP, but he was completely fucked up in his head.

(Nikola, 30-year-old)

Nikola is not precise about his friend's experience with PrEP, nor is there any proven medical evidence concerning the potential side effects of PrEP on the bowel or chronic bowel conditions, but rumors of side effects in general are sufficient for him not to take PrEP for the time being. Aleksandar1, a 34year-old chef, is worried, just like Nikola, about potential side effects of PrEP. He discusses this issue as follows:

PrEP is really bad for your bones. I've heard that PrEP is actually not suitable for people with rheumatoid arthritis.

(Aleksandar1, 34-year-old)

Aleksandar1 is not an isolated example of gay men's concern with the side effects of PrEP. Indeed, the discourse about negative side effects of PrEP among some gay men is a serious obstacle to PrEP's implementation, and this discursive field erodes the potential for the expansion of PrEP as a preventive method against HIV infection.

A further complication impacting the successful introduction of PrEP in Serbia is the price. It is the most significant factor in the acceptance of PrEP among gay men and a key focus of my interviewees' responses when PrEP use was considered. Their answers powerfully illustrate a deep class divide in the Serbian gay community, which impacts public and community health. The context of their answers is an economically weak public health system in Serbia, that does not provide free of charge PrEP. The class divide that is apparent between sub-groups of gay men in Serbia, and its differing impact on the health of those sub-groups, is a serious topic that needs to be foregrounded in a broader 
future research project, focusing on the intersections between sexuality, class and race. We can find a relevant example of precariousness in the testimony of Stefan2, a 23-year-old artist from Belgrade, who thinks that PrEP is expensive. He states as follows:

You can take daily PrEP. The cost is 50 euros per box. I think that's quite expensive. I've heard some rumours that NGOs are lobbying to make PrEP available on prescription for free, but that still hasn't happened.

(Stefan2, 23-year-old)

Stefan2's hope - that PrEP will become available on prescription in the Serbian context - is a longdistance goal, and, at the present moment, it is going to remain out of reach for some time to come. Dragan, a 48-year-old unemployed delivery worker from Belgrade, has the same opinion - that PrEP is expensive and that this is the reason why many gay men are not currently on PrEP:

People say that when they are on PrEP they don't want to use condoms, but when they hear how expensive PrEP is they give up on it.

(Dragan, 48-year-old)

Adherence to PrEP depends on many factors, but one of the main barriers to PrEP use in Serbia is the cost of the drug. In Serbia, the ratio of economic inequality (the difference between the income of the richest and the poorest) is 9:1 and it is one of the widest gaps in Europe (Eurostat, 2018). As discussed above, the pricing and affordability of PrEP is an additional difficulty, given the average monthly salary. The cost of a month's supply of PrEP, which is 50 euros, puts PrEP potentially out of reach for some gay men. Supporting previous claims of PrEP being too expensive is AleksandarH, a 24-year-old waiter, who also cannot consider PrEP use because of the cost. He claims:

You know what you have to pay for PrEP here in Belgrade? The price of 50 euros is absolutely insane!

(AleksandarH, 24-year-old)

Dejan, a 35-year-old hairdresser, also commented on the price of PrEP. He had initially misunderstood and thought that the 50-euro price-tag for PrEP was to purchase enough meds for a threemonth period. He discusses it as follows:

D: The price is ok if it is for protection. When compared with the price of going out, having drinks, shopping, or taxis, that's not too much. None of these are necessities. And with PrEP [...] Is this the price for one-month or for three-month protection? Q: 50 euros is the monthly price.

D: Ahaaa! Ok! You should have told me that detail up front. That's important! Very important! That's too expensive! 
Dejan seemed taken aback when he learned that for 50 euros one could only purchase PrEP for one month and not three months. He insists that such information should be broadly and clearly communicated to the public and that might help people make the decision to take PrEP.

In stark opposition to the previous views of PrEP as an expensive drug, Djordjo, a 25-year-old hairdresser, does not consider PrEP expensive. He comments:

The cost of daily PrEP per month is 50 euros. In my opinion, this is not expensive. (Djordjo, 25-year-old)

Individual economic circumstances - monthly income or lack thereof, level of income, stability/ instability of employment - are markers of class division among gay men, and thus, they are also markers of systemic health inequalities regarding potential HIV prevention. Subsidised PrEP, supported financially by the Public Health Insurance Fund in Serbia, is still hard to imagine and, overall, no prospect of subsidized PrEP in the near future is a contributing factor for the slow introduction of PrEP in Serbia.

Two of my interviewees, Stefan, a 23-year-old artist, and Anonymous2, a 40-year-old physiotherapist, both from Belgrade, discuss the use of PrEP for chemsex purposes. Chemsex is not a novel phenomenon among Serbian gay men (see Milosavljevic, 2017), but the role being played by PrEP in this sexual practice is. This area is clearly under-researched, especially drug-to-drug interactions which could undermine the potential of PrEP. Following this line, an interviewee, Anonymous2, states:

Anonymous2: My ex-boyfriend is HIV positive. I'm aware that HIV transmission happens when drug use is connected with sex.

Q: You mean in Chemsex settings?

Anonymous2: Yes, absolutely.

While Anonymous2 discusses general chemsex practice, Stefan goes further in his testimony. Stefan is not satisfied with the recommendations for PrEP use suggested by the health authorities in Serbia because they deprive chemsex practitioners of PrEP. He elaborates on this topic as follows:

I know that chemsex parties are risky sex environments. You are excluded from being prescribed PrEP in the policy recommendations if you say that you are practicing chemsex. That's discrimination! I don't understand why! We lack proper research on the topic, so we have to draw on case studies from the Netherlands or Germany [...] So, instead of reducing HIV risk, Serbia forbids doctors from prescribing PrEP use for chemsex. That's such a shame! I've heard that doctors are afraid that if PrEP use is inconsistent, then the virus could mutate and that's why they ban chemsex users from getting PrEP on prescription. I disagree with that! (Stefan, 23-year-old) 
Here, Stefan discusses important issues regarding the use of PrEP in Serbia. Stefan claims that, in relation to PrEP, health practitioners discriminate against certain sexual practices amongst gay men: some sexual practices are deserving of PrEP and some like chemsex are not. On the part of the medical professionals, fear of PrEP misuse and consequently, of the risk of the growth of viral resistance to ARVs, is driving such decision-making. In order to enable as many PrEP users as possible to access PrEP, the development of national PrEP policy should be much more inclusive. A truthful account of the whole gamut of different sexual practices in the community of gay men and MSM should be the focus of HIV prevention policy and that must include chemsex. Castro, Delabre and Molina (2019) emphasize that "risk compensation remains a frequent argument against the availability and provision of prevention methods for vulnerable populations." Although the question of improper use of PrEP (adherence) and potential viral resistance to ARVs are legitimate issues, those issues should be researched far more thoroughly in the Serbian context. The acquisition and the use of PrEP in Serbia shows the lack of clear public health guidelines as well as an unregulated market. There is a need to update PrEP-use protocols to be in line with ECDC guidelines. Most of PrEP in Serbia is sourced on the black market, which indicates that public health services should take this into account when developing any strategies for HIV prevention.

The use of PrEP in Serbia is not on recommendation from medical professionals to gay men. Rather, gay men amongst themselves recommend and self-regulate the use of PrEP. Their decisions in turn draw on the following factors: stigma in relation to PrEP use, the disclosure of HIV status, and risky sexual behaviour. Such recommendations also create a tension between HIV positive and HIV negative gays in the community.

Recommending PrEP to others is clearly connected to another issue - an HIV health identity and its disclosure. You can recommend PrEP only to those gay men who are HIV negative, and this leads to a paradoxical mismatch of identity. The recommendation of PrEP to others was clearly a very divisive issue among my interviewees and they responded as follows - 16 said they would recommend PrEP to others, 4 would not recommend it, and 10 were not sure whether they would recommend it or not.

Milos2, a 33-year-old unemployed waiter from the outskirts of Belgrade, discusses this issue as follows:

M2: Yes, I know of PrEP. I saw many men advertise themselves on Grindr or Romeo saying they are on PrEP. The most frequent users of PrEP are foreigners when they come to visit [...] Local boys say they are on PrEP, but at the same time, they are HIV positive.

Q: What do you mean? How is that possible?

M2: Well [...] I don't know, but that's what they say. They think that if they are HIV positive and they take ARV then they are on PrEP, too. It's the same thing for them. 
Milos2 discusses an interesting phenomenon that he spotted on gay dating websites. For some gay men any treatment regime involving the use of ARVs is equated with PrEP use, and this conflation of the two is then incorporated into gay online identity and (re)presentations. A novel identity emerges online - "undetectable, on PrEP." In online gay communication, some gay men who are HIV positive consider themselves more attractive to others if they say they are on PrEP, instead of saying they are on ARV, because they think it will imply that they are HIV negative, instead of HIV positive. In this context, they believe that an undetectable viral load, as the result of treatment with ARVs of an already existing infection, is the same as PrEP's protective use - i.e. the effect is that the virus is blocked, thus un-transmittable. As Race summed up this situation, it is "how things must appear and be experienced by those of different sero-status" (2016: 18).

Among gay men, the self-designated category "on PrEP" is extended not only to those who are HIV negative and on PrEP, but also to those who are HIV positive and on ARV with an undetectable viral load - "undetectable, on PrEP" category. The result of the traumatic effects of HIV/AIDS epidemic, the health paradigm and the premise "life is easier when you are not HIV positive" is firmly reflected in this gay men's identity in Serbia. It can clearly be seen in this study that the true HIV status of respondents is being disregarded and PrEP is being judged to be used as a pharmaceutical "prosthesis," making viable a significant change in gay subjectivity - a transformation of HIV positive identity on ARV into an HIV negative one on PrEP. As discussed, this shift has a repercussion on risk assessment in sexual practices. In the mind of some HIV positive gay men, if PrEP use equals antiretrovirals use, then this could reverse the position of risk among gay men in a following way:

By comparison, PrEP asks HIV-negative men to confront the structure of exception head on, as it were: to identify themselves as subjects of risk in the mode of precalculation and intentionality. Perhaps, then, PrEP is such a reluctant object partly because it makes explicit something that is difficult to be explicit about from within one of the common orientations to sex and risk among gay men today: the desire to position risk as an exception rather than a tendency, a 'straying afield of oneself' rather than something as coherent or culpable as a habit or a pre-calculated decision.

(Race, 2016: 24-25)

In a metaphorical "equation," HIV positive gay men on ARV achieve immunological equilibrium with HIV negative gay men on PrEP. Preciado (2013) discusses the importance of "immunological equilibrium" as lying at the heart of subjectivity in what he calls the "pharmacopornographic era" the era when biomedical interventions and pharmaceutical technologies (and PrEP is the one of them) transform someone's identity. Due to this power of transformation PrEP use is seen as a valuable addition to the available potency of antiretroviral medicines (ARV) to prevent HIV transmission and to establish immunological balance in the body. Following the same logic, prevention of HIV transmission is the result of successful ARV therapy based on the principle " $U=U$ " or "unde- 
tectable=untransmittable" (Vernazza et al., 2008). In this respect, the Serbian context is not an exception from the rule. In Canada, Grace et al. (2018) discuss an interesting phenomenon among some Canadian gay men who believe PrEP use to be a responsible pharmacological method to achieve an undetectable viral load.

The exclusive case for PrEP-induced HIV protection is confirmed by two of my interviewees, both of whom consider PrEP as an extreme measure that enables people to proceed to have sex without concern for the potential infectiousness of a sex partner in the "heat of the moment" situations. For them, HIV status is erased from the identity of potential sex partner(s) and is not considered relevant in risky situations since "PrEP on demand" allows them to proceed freely to the realization of sexual desires. A good example of this is Senad, a 50-year-old salesman, who discusses PrEP use as follows:

I would take PrEP when the guy is HIV positive and in circumstances where I'm hot for him and I want to have sex with him. I don't like to use condoms. Sex is much easier without them. HIV infection is not a 'bogey man' anymore.

(Senad, 50-year-old)

This situation of PrEP "on demand" described by Senad reflects the extent to which the perception of HIV prevention is completely transformed somehow in line with Race, as "escaping the pressure of the condom imperative" (2016: 24). Sharing the same thoughts as Senad is Nino, a 50-year-old salesman from Belgrade, who states as follows:

The use of PrEP is very logical. I would take it if I want to prevent HIV when I like someone irresistibly. Fuck, let's do it! That's it, I would use it when I do not have info on someone's HIV status and I want to have sex with him right now.

(Nino, 50-year-old)

Nino and Senad both link the "heat of the moment" situations with rejection of condom use and both consider PrEP use as a form of protection against HIV. The PrEP protocol in Serbia is unfortunately insufficient as it lacks the possibility for PrEP "on demand" use. Officially, the only possible way to use PrEP is a continuous use and this is the reason why most gay men turn to the black market to access PrEP. They can get PrEP when it suits them, before sex or before events where they expect to find sexual partner(s). The fear of potential misuse or non-adherence to PrEP by medical professsionals is grounded as it leads to HIV viral resistance to ARV drugs.

Online user profiles with the designation "on PrEP" have a highly affirmative impact in online gay communication in Serbia. The gay community on gay dating sites in Serbia frequently includes PrEP on their profiles. Nikola2, a 23-year-old medical student, discusses an interesting phenomenon which he has spotted online: 
When you communicate with them online, everyone is on PrEP. I simply don't believe it! Look [...] (Nikola2 is showing to me his phone screen and a user profile from Grindr. There is a picture of a man's torso with the caption: "On PrEP") You see? So many men claim to be on PrEP and yet it is still so underused. That's our reality [...] Everyone says they are on PrEP, and they are not (laughs) [...] I do not believe they take PrEP at all!

(Nikola2, 23-year-old)

The arm's-length nature of virtual communication and online anonymity allows for virtual "playing" with HIV status and health identity among gay men even in the time of the undetectable viral load. What my interviewees said puts into question the truthfulness of those posting the PrEP info on their online profiles. This was most evident when they discussed PrEP use and sex in risky collective environments. The following example is a vivid illustration of such a situation. AleksandarH, a 24 years old waiter, discuses misuse of PrEP:

I prefer condoms, but when I went to this sex party, no one was talking about PrEP. There was no protection policy at all! I went there and I escaped. I saw 5 or 6 guys fuck with one guy [...] In my opinion, I think that PrEP abuse is very much our reality. I've heard that foreigners bring PrEP to Belgrade and when they organise parties here, they give PrEP to our boys. Just one pill and that's it! The boys believe they are protected because someone says so. Imagine, no risk for them at all! (AleksandarH, 24-year-old)

AleksandarH discusses the risk of HIV as inherently based on shared responsibility among potential sex partners, which is mostly absent in collective sex environments. In this context, the loss of control in sex and inadequate PrEP use lead to unsuccessful HIV prevention due to suboptimal dosage and non-adherence to PrEP. For AlexandarH, the abuse of PrEP relates to the casual disregard by potential users of the need for strict adherence to a specific PrEP use regime if it is to be effective as preexposure prophylaxis against HIV infection. Changing this situation is going to require far more positive attitudes to sex and intimacy in the gay community. If the use of PrEP is to increase to a level where its potential for protection among gay men is realised, such positive attitudes to sex and intimacy need to be built into promotion campaigns for PrEP (see Keene et al., 2020).

\section{PrEP use and stigma reproduction in Serbia}

Different forms of stigmatization relate to the gay identity and PrEP, and they not only intertwine with each other, but they also have a cumulative effect on the dismissal of PrEP as a positive force, sometimes even contributing to the PrEP paradox. All forms of stigmatization undermine the implementation and protective potential of PrEP. In 2012, when PrEP first became available in the the US, PrEP users were named "Truvada whores" because they were regarded as more promiscuous and more risk-taking in sex. This type of stigma is well documented (Duran, 2012; Calabrese and Underhill, 
2015; Haire, 2015; Jaspal and Daramilas, 2016; Spieldenner, 2016; Eaton et al., 2017; Hildebrandt, Bode and $\mathrm{Ng}, 2019$ ). Spieldenner (2016) describes the phenomenon of "slut shaming" directed towards PrEP users. Furthermore, Dubov et al. (2018) discuss stereotyping as a form of stigma and they relate this to PrEP-use based on "stereotypes of promiscuity, chemsex, condomless sex, or sex work" (p. 1835).

The stigma related to gay sexuality affects all aspects of gay lives, including HIV treatment and prevention, and Serbia proves to be no exception to the rule (Milosavljevic, 2012; 2017). The interviewees in my research confirmed the influence of stigma on PrEP access, as 6 out of 30 interviewees (20\%) would not ask their GP for a prescription for PrEP. 3 out of 6 state the reasons for this decision as follows: first, they do not see themselves as promiscuous (1 interviewee); second, they could not bear the "hassle" of going to see their GP, a fact that is can be attributed to a hidden fear of enforced coming out (1 interviewee), and third, a preference for the use of condoms over PrEP protection (1 interviewee). 3 interviewees out of 6 claimed that they would not go for the PrEP prescription and PrEP regime because they feared being stigmatized in/by the medical institution (which accounts for $50 \%$ of those who would not go to see their GP for the PrEP prescription, and $10 \%$ of the overall number of interviewees involved in the research). Igor, a 33-year-old economist from Belgrade, explains his decision not to get PrEP on prescription in this extract from his interview:

I: I wouldn't go to see my GP to get a prescription for PrEP. I would feel embarrassed because doctors are biased - they gossip about who's gay and I find the gossip intolerable. You have to go through that over and over again.

Q: You think if you accepted a prescription, you would be forced to come out to your GP?

I: Absolutely! Their reaction to homosexuality is hideous - they don't know anything about sexual diversity. Do you really think that my GP wouldn't ask me - why do you need PrEP? What do you need it for? They are so narrow-minded.

Q: So, in your opinion, you would feel stigmatised if you approached your GP and asked to be prescribed PrEP?

I: Of course I would! Medical professionals lack the knowledge about the needs of gay men and disregard the problems which they face because of their sexuality in everyday life. Medical doctors are ignorant about that.

All sexual minorities are persistently stigmatized in Serbia and this also holds true throughout the medical professions and institutions (see Kocić et al., 2008; Vowa et al., 2015). Igor's argument about the stigmatisation of gay men is proof that such a stigma affects the overall body politic of gay men in Serbia. It certainly affects Igor's decision not to get PrEP and it deepens his internalized stigma. Dragan, a 48-year-old delivery man, and AleksandarH, a 24-year-old waiter give similar answers. Dragan states that stigmatization affects his decision to take the PrEP prescription. 
If you want to get a prescription for PrEP from your GP, you have to tell him or her that you are gay and that's the problem (Dragan mimes looking uncomfortable and pulls his face into a grimace).

(Dragan, 48-year-old)

Stigma becomes internalised in the minds of gay men in Belgrade who are potential PrEP users, and we can see how it operates in the way AleksandarH thinks about medical doctors, PrEP and gay men. He elaborates more fully on the matter, stating that he would not dare to go to see his GP to be prescribed PrEP. He would not like to be forced to come out in a GP's office and he fears the judgement of doctors:

Medical doctors have a really bad approach. They are simply judgmental! They stigmatise! [...] I wouldn't dare to go to them to sort out a prescription. They don't understand gay men. They only judge.

(AleksandarH, 24-year-old)

A substantial number of the gay men involved in the study, as many as 7 out of 30 (23.3\%), express the fear that they would be stigmatised in medical institutions by medical professionals. This is an obstacle to biomedical HIV prevention and it is not without significance when it comes to how to improve both overall HIV preventive methods and improved adherence to the PrEP regime. The introduction and implementation of PrEP in Serbia reveals a systemic social stigmatisation of gayness which, if not checked, could significantly reduce the access to PrEP provided by the state and consequently undermine HIV preventive programs.

Some other forms of stigma related to PrEP also appear to be highly significant. When we consider the recommendation of PrEP within the gay community, some of my interviewees see PrEP as intended exclusively for those who are "very promiscuous." Thus when respondents were asked to whom they would recommend PrEP, this "very promiscuous" group was the first group that came to their mind. Golub, Gamarel and Surace (2017) discuss "promiscuity" as the most common form of stereotyping of PrEP users among gay men. Anonymous2, a 40-year-old physiotherapist from Belgrade, claims:

Yes, I would recommend PrEP to highly promiscuous men. For instance, sex tourists, no matter gay or straight, are the ones who are supposed to take it! That's because HIV is still here and those who get it are not monogamous men, but highly promiscuous.

(Anonymous2, 40-year-old)

The stigma that surrounds PrEP use is present in the discussion among my interviewees and the response of Anonymous2 follows this line of thought when PrEP use is discussed. In the group of 
respondents who consider PrEP use as exclusively relevant to "highly promiscuous" men is Aleksandar, a 33-year-old artist, who states:

I don't know who should take PrEP. I think it's your character that defines those who are supposed to take it. The uptake depends on the number of sex partners you have.

(Aleksandar, 33-year-old)

There was no consensus among my interviewees as to the definition of "highly promiscuous men." Neither did they agree on how many sex partners - past and/or present - were necessary to qualify as promiscuous. The category "promiscuous" is individually and arbitrarily constructed and does not provide a clear-cut measure of when/at what point/whether you are considered promiscuous or not. Nevertheless, the discourse of promiscuity among gay men in Serbia certainly erodes the potential of PrEP to prevent further HIV transmission, as many gay men do not want to be labeled "promiscuous."

\section{Conclusions}

This article explored some paradoxes regarding PrEP use in Serbia that range from the rejection of and reluctance to use PrEP to the novel forms of gay sex/health identity that result from PrEP use. The biomedical HIV prevention in the form of PrEP is a signifier of how the new technological regulation of HIV influences homosociality, gay online communication, sexual practices, and stigmatization. Although PrEP is considered to be designed for HIV prevention by HIV negative gay men, some HIV positive men are willing to express their belief that ARV therapy is the same thing as PrEP. They form and use a new identity position - "undetectable, on PrEP" - and through this process gay men switch from "HIV positive" or "undetectable on ARV" to "on PrEP" position. This novel identity has repercussions in online gay communication and representation, but it also impacts risk assessment and sexual practices.

Since the introduction of PrEP in Serbia in August 2019, the uptake of PrEP has been very low. The current PrEP uptake among gay men in Belgrade and Serbia could be described as still "informal" and "event-based." In 2020, there was no significant improvement in the accessibility and availability of PrEP. Since the protocol for PrEP use in Serbia is restrictive, PrEP "on demand" or "event-driven" PrEP is still not officially incorporated in the policy.

There is a huge gap between the objective risk of HIV and the subjective perception of "risk-whileon-PrEP" among gay men. The relation between PrEP and HIV status has been communicated as a paradox on gay dating apps and websites in tandem with the emergence, in gay online communication, of the new hybrid identity "on PrEP" or "undetectable, on PrEP." New information and knowledge gained from gay men's practices and experiences with PrEP should inform lesson-learning and be widely incorporated into the policy on PrEP in Serbia. Promotional activities regarding PrEP use in 
Serbia should focus on the access and availability of PrEP, but also on the necessity to emphasize who qualifies for PrEP use and who is on ARV therapy. What is more, the difference between ARV therapy and PrEP should be clearly communicated by the health authorities. The information that ARV is for HIV-positive persons and PrEP is for HIV-negative persons should be easily accessed and spread in the gay community.

PrEP as a prevention tool has a target group of HIV negative gay men. It is meant to be for HIV prevention and not for the treatment of HIV infection. Sometimes, gay men who are on antiretroviral therapy claim that they are on PrEP. In doing so, they falsely present themselves as HIV negative. Although an undetectable HIV viral load status means no HIV transmission, it is not the same as HIV negative status. Thus, the tension between HIV negative and HIV positive gay men in the gay community in Serbia has entered a new phase of non-trust.

Making recommendations to others that they should consider PrEP use is proof that PrEP is an accepted and trustworthy tool for risk reduction. It shows confidence in someone's HIV status and consistent risk assessment. Nevertheless, the stigma which surrounds PrEP use, reluctancy and rejection of it, as well as HIV status mismatch in relation to PrEP use are all obstacles to successful HIV prevention in Serbia. This article is intended to initiate a debate on the biomedical prevention of HIV through PrEP, and its implications in the context of Serbia.

\section{Works Cited}

Auerbach, Judith D. and Trevor A. Hoppe (2015), "Beyond 'getting drugs into bodies': social perspectives on pre-exposure prophylaxis for HIV", Journal of International AIDS Society, 18.3: 19983. https://doi.org/10.7448/IAS.18.4.19983

Brisson, Julien (2017), "Ethical public health issues for the use of informal PrEP", Global Public Health: An International Journal for Research, Policy and Practice, 13.10: 1382-1387. https://doi.org/10.1080/17441692.2017.1373139

Brennan, Joseph (2017), "Gay Porn's Bareback Momentum", Journal of Homosexuality, 67.1: 127157. https://doi.org/10.1080/00918369.2018.1525947

Calabrese, Sarah K. and Kristen Underhill (2015), "How stigma surrounding the use of PrEP undermines prevention and pleasure: A call to destigmatize 'Truvada Whores', American Journal of Public Health, 105.10: 1960-1964. https://dx.doi.org/10.2105\%2FAJPH.2015.302816

Castro, Daniela R., Rosemary M. Delabre and Jean-Michael Molina (2019), "Give PrEP a chance: moving on from the 'risk compensation' concept", Journal of the International AIDS society, 22.S6: 50-56. https://doi.org/10.1002/jia2.25351

Dean, Tim (2015), "Mediated intimacies: Raw sex, Truvada, and the biopolitics of chemoprophylaxis", Sexualities, 18.1/2: 224-246. https://doi.org/10.1177\%2F1363460715569137 
Dubov, Alex, et al. (2018), "Stigma and Shame Experiences by MSM Who Take PrEP for HIV Prevention: A Qualitative Study", American Journal of Men's Health, 12.6: 1843-1854. https://doi.org/10.1177\%2F1557988318797437

Duran, David (2012), "Truvada Whores?", Huffington Post Gay Voices, http://www.huffingtonpost.com/david-duran/truvada-whores_b_2113588.html

Eaton, Lisa A., et al. (2017), "Stigma and Conspiracy Beliefs Related to Pre-exposure Prophylaxis (PrEP) and Interest in Using PrEP Among Black and White Men and Transgender Women Who Have Sex with Men", AIDS and Behavior, 21: 1236-1246. https://doi.org/10.1007/s10461-017$1690-0$

Grace, Daniel, et al. (2018), "The Pre-Exposure Prophylaxis (PrEP)-Stigma Paradox Learning from Canada's First Wave of PrEP Users", AIDS Patient Care and STDs, 32.1: 24-30. https://doi.org/10.1089/apc.2017.0153

Golub, Sarit A., Kristi E. Gamarel and Anthony Surace (2017), "Demographic Differences in PrEPRelated Stereotypes: Implications for Implementation", AIDS Behaviour, 21: 1229-1235. https://doi.org/10.1007/s10461-015-1129-4

Gomez, Walter, et al. (2020), "PrEP uptake as a social movement among gay and bisexual men", Culture, Health \& Sexuality, 29: 1-13. https://doi.org/10.1080/13691058.2020.1831075

Haire, G. Bridget (2015), "Preexposure prophylaxis-related stigma: strategies to improve uptake and adherence - a narrative review", HIV/AIDS Research and Palliative Care, 7: 241-249. https://doi.org/10.2147/HIV.S72419

Hildebrandt, Timothy, Leticia Bode and Jessica S. C. Ng (2020), "Responsibilization and sexual stigma under austerity: Surveying public support for government-funded PrEP in England", Sex Res Soc Policy, 17: 643-653. https://doi.org/10.1007/s13178-019-00422-z

Institute of Public Health of Serbia - IOPHOS (2020), Godišnji izveštaj o zaraznim bolestima u Republici Srbiji za 2019. godinu [Annual report on communicable diseases for 2019], www.batut.org.rs/index.php?content $=2241$

Jaspal, Rusi and Christos Daramilas (2016), "Perceptions of pre-exposure prophylaxis (PrEP) among HIV-negative and HIV-positive men who have sex with men (MSM)", Cogent Medicine, 3.1: 1256850, https://doi.org/10.1080/2331205X.2016.1256850

Keene, Lance, et al. (2020), "\#PrEP4Love: success and stigma following release of the first sexpositive PrEP public health campaigns", Culture, Health \& Sexuality, 23.3: 397-413. https://doi.org/10.1080/13691058.2020.1715482

Klein, Hugh and Thomas Alex Washington (2020), "Why more men who have sex with men are not using PrEP-The role played by lack of interest in learning more about PrEP", Journal of Gay \& Lesbian Social Services, 32.1: 99-114. https://doi.org/10.1080/10538720.2019.1681339

Kocić, Biljana et al. (2008), "Professional risk, knowledge, attitudes and practices of health care personnel in Serbia with regard to HIV and AIDS", Central Europe Journal of Public Health, 16.3: 134-137. https://doi.org/10.21101/cejph.a3470

Milosavljević, Zoran (2012), AIDS and its discontents in Serbia: Silencing gay sexuality in the age of illness, unpublished MA thesis, University of Hull/UK, University of Lodz/Poland, https://www.researchgate.net/publication/271136305_AIDS_and_Its_Discontents_in_Serbia_Sile ncing_Gay_Sexuality_in_the_Age_of_Illness 
Milosavljević, Zoran (2017), "My happiness is your horror: discourses of virtual communication and HIV/AIDS in gay men's narratives in Serbia", Journal of Gender Studies, 26.1: 23-32. https://doi.org/10.1080/09589236.2016.1266998

Nicholls, Jay Emily and Marsha Rosengarten (2019), "PrEP (HIV pre-exposure prophylaxis) and its possibilities for clinical practice", Sexualities, 23.8: 1327-1342. https://doi.org/10.1177/1363460719886556

Preciado, Paul (2013), Testo Junkie: Sex, Drugs, and Biopolitcs in the Pharmacopornografic Era, New York, Feminist Press.

Race, Kane (2016), "Reluctant Objects: Sexual Pleasure as a Problem for HIV Biomedical Prevention", GLQ A Journal of Lesbian and Gay Studies, 22.1: 1-31. https://doi.org/10.1215/106426843315217

Spieldenner, Andrew (2016), "PrEP Whores and HIV Prevention: The Queer Communication of HIV Pre-Exposure Prophylaxis (PrEP)", Journal of Homosexuality, 63.12: 1685-1697. https://doi.org/10.1080/00918369.2016.1158012

Statistical Office of the Republic of Serbia (2021), Average net salaries and wages in the Republic of Serbia - October 2021.

www.stat.gov.rs/enus/vesti/statisticalrelease/ $p p=8395 \& a=24 \& s=2403 ? s=2403$

Thomann, Matthew (2018), "'On December 1, 2015, sex changes. Forever': Pre-exposure prophylaxis and the pharmaceuticalisation of the neoliberal sexual subject", Global Public Health, 13.8: 997-1006. https://doi.org/10.1080/17441692.2018.1427275

UNAIDS (2020), Country factsheets Serbia, www.unaids.org/en/regionscountries/countries/Serbia

Vernazza, Pietro et al. (2008), "HIV transmission under highly active antiretroviral therapy", The Lancet, 372.9652: 1806-1807. https://doi.org/10.1016/S0140-6736(08)61753-5

Vowa, E.M., Janković, J. and T. Savu (2015), "Knowledge, attitude and behaviour of Belgrade medical students related to HIV/AIDS", European Journal of Public Health, 25.3: 436.

https://doi.org/10.1093/eurpub/ckv176.169 